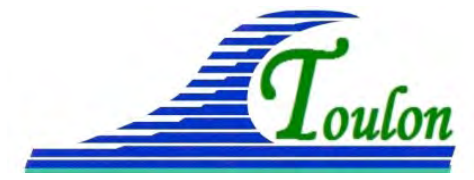
XIV $V^{\text {èmes }}$ Journées Nationales Génie Côtier - Génie Civil
Toulon, 29 juin au $1^{\text {er }}$ juillet 2016

DOI:10.5150/jngcgc.2016.080 (C) Editions Paralia CFL

disponible en ligne - $h$ ttp://www.paralia.fr - available online

\title{
Prise en compte de la dispersion fréquentielle pour la simulation des tsunamis trans-pacifique : le cas des tsunamis chiliens de 2010 et 2015
}

\author{
Adrien POUPARDIN ${ }^{1}$, Philippe HEINRICH ${ }^{1}$, Hélène HEBERT ${ }^{1}$, \\ Anthony JAMELOT ${ }^{2}$, Dominique REYMOND ${ }^{2}$, François SCHINDELE ${ }^{1}$
}

\author{
1. CEA DAM DIF, F-91297 Arpajon Cedex, France. \\ adrien.poupardin@cea.fr \\ 2. CEA DASE, BP 640, 98713 Papeete, Tahiti.
}

Résumé : La propagation trans-Pacifique du tsunami chilien de 2010 est simulée en résolvant soit les équations de Saint-Venant soit celles de Boussinesq, ces dernières prenant en compte la dispersion fréquentielle des vagues. Les résultats numériques indiquent que la modélisation de la dispersion fréquentielle est nécessaire pour reproduire les hauteurs d'eau enregistrées par différentes bouées DART du Pacifique.

Indépendamment du modèle choisi, la résolution des grilles utilisées modifie significativement la forme du tsunami modélisé. Des tests de sensibilité monodimensionnels montrent que le modèle de Boussinesq converge à partir d'un pas d'espace de $5 \mathrm{~km}$ et que le modèle de Saint-Venant, utilisé avec un pas d'espace de 10 $\mathrm{km}$, génère de la dispersion numérique proche de la dispersion fréquentielle physique.

Enfin, l'analyse des spectrogrammes montre que la dispersion fréquentielle du tsunami a un impact non négligeable sur le temps d'arrivée, l'amplitude et la fréquence des vagues se propageant en champ lointain. Elle doit donc être prise en compte pour l'étude de l'amplification des vagues lorsque celles-ci atteignent les côtes, et notamment dans les zones habitées à risque.

Mots clés : Dispersion fréquentielle, Tsunami, Simulation numérique, Bathymétrie.

\section{Introduction}

La dispersion fréquentielle des tsunamis se traduit par la formation d'un train d'ondes pour lequel les ondes longues se propagent plus rapidement. Traditionnellement, cette dispersion n'est pas prise en compte pour des tsunamis générés par des séismes de forte magnitude. Ces tsunamis sont en général formés d'une seule vague dont la longueur d'onde est suffisamment grande par rapport à la profondeur pour se propager en champ lointain sans se disperser. L’importance de la dispersion est généralement admise pour les événements d'origine tectonique de magnitude inférieure ou égale à 8 ou pour les tsunamis issus de sources gravitaires de petites dimensions. C'est notamment le cas du séisme de 2009, de magnitude 8.1, à l'extrémité nord de la faille de Tonga qui a généré un tsunami dispersif ayant conduit à la formation d'un train d'ondes affectant les îles voisines des Samoa (ZHOU et al., 2012). Pour des séismes plus forts, la dispersion 


\section{Thème 7 - Risques côtiers}

fréquentielle se manifeste également mais à une distance plus importante de la source. Ainsi, suite au séisme de Sumatra en 2004 (de magnitude 9.1), un train d'ondes a été observé dans la baie de Bengal et sur la mer Andaman (IOUALALEN et al., 2007 ; HORRILLO et al., 2006). De même, le tsunami de Tohoku en 2011 (de magnitude 9) a été affecté par la dispersion fréquentielle en champ lointain (LØVHOLT et al., 2012, GRILLI et al., 2012), au-delà de $10000 \mathrm{~km}$ de la source. Une étude paramétrique a été menée par GLIMSDAL et al. (2013) afin de déterminer un temps d'apparition de la dispersion qui dépend principalement des dimensions de la source (séisme ou glissement de terrain) et de la profondeur d'eau.

Dans le cas des tsunamis chiliens d'origine tectonique de 2010 et 2015, la dispersion fréquentielle affecte les signaux enregistrés par différentes bouées DART et marégraphes du Pacifique (SAITO et al., 2010). Cette dispersion fréquentielle peut être simulée en champ lointain (LØVHOLT et al. 2008, ZHOU et al., 2011) en considérant les équations de Boussinesq à condition de disposer d'une puissance de calcul importante. Le modèle de Saint-Venant peut également être utilisé à condition que la dispersion numérique induite par ce modèle se rapproche de la dispersion fréquentielle physique (ZHOU el al., 2012). Dans ce cas particulier, les résultats obtenus en utilisant les équations de Saint-Venant sont acceptables par rapport aux observations et aux résultats obtenus en utilisant les équations de Boussinesq. L'utilisation de ce système d'équations, hors de son domaine de validité, n'a de sens que d'un point de vue opérationnel car, dans ce cas particulier, les temps de calcul doivent être suffisamment courts pour évacuer les populations des zones à risque et ceci très rapidement.

Les études de sensibilité menées par le centre polynésien de prévention des tsunamis montrent qu'une incertitude de quelques degrés sur l'azimut du plan de faille modifie significativement les prévisions de hauteurs de vague sur les côtes de la Polynésie Française (REYMOND et al., 2013; JAMELOT \& REYMOND, 2015). Nous proposons de la même manière de montrer que la prise en compte de la dispersion fréquentielle pour la simulation des tsunamis doit permettre d'améliorer sensiblement la prévision du temps d'arrivée et de la hauteur des plus grosses vagues atteignant les baies amplificatrices des îles du Pacifique.

\section{Méthodologie}

Les tsunamis chiliens de 2010 et de 2015 ont été simulés par les modèles de SaintVenant et de Boussinesq. Seuls les résultats de 2010 sont présentés dans cette note. Le tsunami de 2010 a fait l'objet de nombreuses études dans la littérature et peut être considéré comme un cas de référence (SAITO et al., 2010). La source de Hayes (NEIC, see http://earthquake.usgs.gov) a été retenue pour nos simulations dans la mesure où les signaux calculés semblent les plus proches de ceux observés, indépendamment du modèle choisi. 


\section{XIV èmes Journées Nationales Génie Côtier - Génie Civil \\ Toulon, 29 juin au $1^{\text {er }}$ juillet 2016}

\subsection{Les grilles utilisées}

Dans un premier temps, la simulation numérique du tsunami chilien de 2010 est réalisée dans une seule grille représentée figure 1 .

Elle n'utilise donc pas de grilles imbriquées pour simuler les phénomènes d'amplifications à la côte.

Les résolutions de grille utilisées sont les suivantes : $2 \mathrm{~min}, 5 \mathrm{~min}$ et $10 \mathrm{~min}$. Ce paramètre a un impact important sur la directivité du tsunami et induit de la dispersion numérique lorsque la résolution se dégrade (ZHOU et al., 2012).

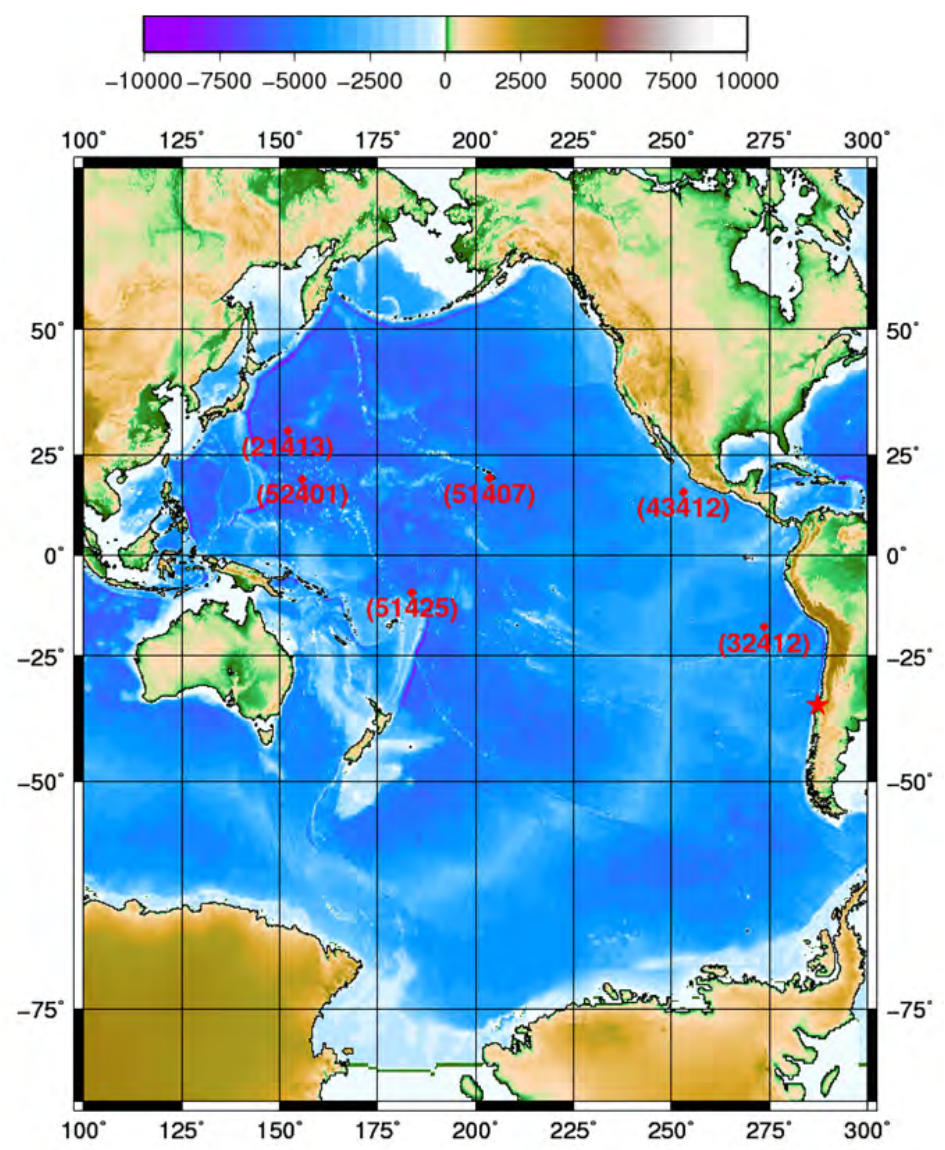

Figure 1. Grille de simulation sur la zone Pacifique : la localisation des bouées DART est indiquée par les losanges rouges, la position du séisme est indiquée par l'étoile rouge (source USGS).

\subsection{Les données utilisées}

Les résultats de nos simulations sont comparés aux signaux issus de plusieurs bouées DART sélectionnées (DART 32412, 43412, 51407, 51425, 52401 et 21413) afin de pouvoir suivre le tsunami dans sa propagation.

Ces derniers signaux sont filtrés afin de faire disparaître les oscillations basses fréquences induites par la marée. 


\section{Thème 7 - Risques côtiers}

\subsection{Les codes de simulation numérique}

Les codes de simulations numériques permettent de simuler le tsunami en résolvant : (1) les équations de Saint Venant qui considèrent les vagues suffisamment longues par rapport à la profondeur pour ne pas être affectées par la dispersion fréquentielle, (2) les équations de Boussinesq qui prennent en compte les phénomènes de dispersion fréquentielle. Les simulations réalisées sont décrites dans le tableau 1.

Tableau 1. Description des simulations réalisées : (1) équations de Saint Venant, (2) équations de Boussinesq.

\begin{tabular}{lll}
\hline Simulations & Système d'équations & Résolution de la grille \\
\hline S1 & $(1)$ & $10 \mathrm{~min}$ \\
S2 & $(1)$ & $5 \mathrm{~min}$ \\
S3 & $(1)$ & $2 \mathrm{~min}$ \\
S4 & $(2)$ & $2 \mathrm{~min}$ \\
\hline
\end{tabular}

\subsubsection{Discrétisation spatiale}

Les équations sont résolues par une méthode de différences finies dans une grille de type C (au sens d'Arakawa), qui permet de conserver «naturellement » le volume dans une maille : les hauteurs d'eau sont calculées au centre des mailles et les vitesses sur les bords de la maille. Pour minimiser la dissipation, la discrétisation des dérivées spatiales des termes linéaires utilise un schéma centré. Seuls les termes d'advection sont discrétisés par un schéma décentré amont, afin d'éliminer d'éventuelles oscillations numériques dans le cas de non linéarités importantes.

Le modèle de Boussinesq utilise une méthode de splitting séparant les directions $\mathrm{x}$ et $\mathrm{y}$ pour résoudre les équations du mouvement, contrairement au modèle de Saint-Venant qui résout les deux équations simultanément. Plus précisément, les deux équations du mouvement sont résolues par un schéma ADI (Alternating Direction Implicit), où les termes dans l'une des deux directions sont traités de façon implicite, tandis que les termes dans l'autre direction sont traités explicitement. Le principal intérêt de cette discrétisation est d'écrire le système d'équations sous forme matricielle, où toutes les matrices sont tri-diagonales et inversées par une méthode de type LU.

\subsubsection{Discrétisation temporelle}

Les équations de continuité et du mouvement sont discrétisées par la méthode de CrankNicolson. La discrétisation de l'équation de continuité et du mouvement par ce schéma conduit à des équations implicites. La structure obtenue est à diagonale dominante et permet d'utiliser une méthode itérative de point fixe. Le test de convergence est effectué sur l'équation de continuité afin de conserver rigoureusement le volume d'eau.

Dans le cas de vagues de faible amplitude, les termes non-linéaires sont négligeables vis-à-vis des autres termes qui sont discrétisés par un schéma centré en temps et en espace. Les termes dominants dans l'erreur de troncature font donc intervenir des 


\section{XIV ${ }^{\text {èmes }}$ Journées Nationales Génie Côtier - Génie Civil \\ Toulon, 29 juin au $1^{\text {er }}$ juillet 2016}

dérivées impaires (des dérivées troisièmes), ce qui conduit à une dispersion numérique dominante vis-à-vis de la dissipation.

\section{Résultats}

Les résultats des simulations sont présentées figure 2 dans un ordre chronologique (temps d'arrivée du tsunami des bouées DART les plus proches aux plus lointaines).

Le modèle utilisé induit un décalage temporel sur la propagation des vagues car certains phénomènes physiques ne sont pas pris en compte dans la modélisation. Ces décalages en temps agissent principalement sur les vagues de périodes supérieures à $1000 \mathrm{~s}$ et sont dus à d'autres formes de dispersion, induites par la compressibilité de l'eau, par l'élasticité de la terre et par les variations de gravité induites par l'ellipticité de la terre (WATADA et al., 2014).
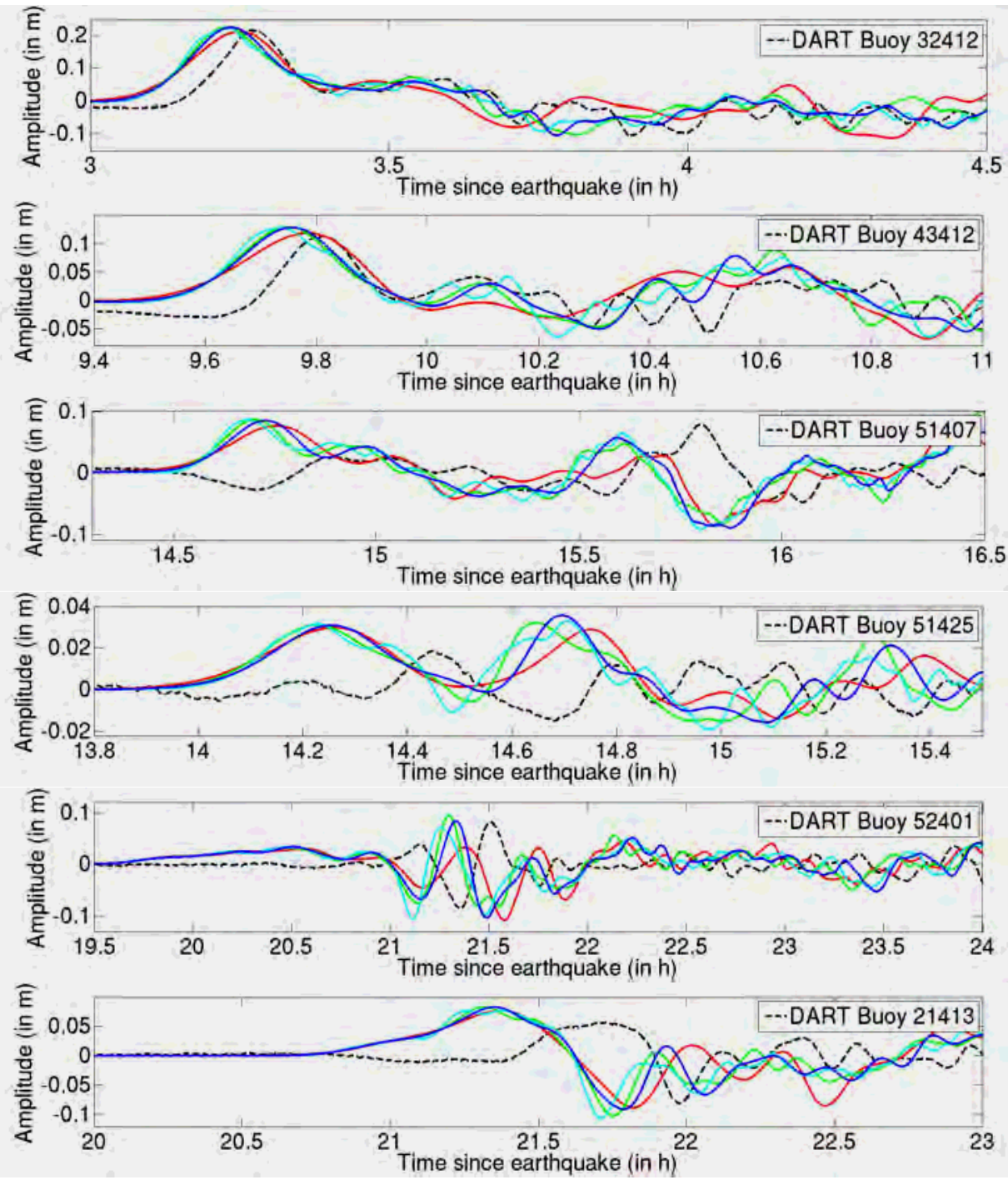

Figure 2. Signaux mesurés et simulés pour les bouées DART considérées : (---) signal mesuré, (-) simulation S1, (-) simulation S2, (-) simulation S3, (一) simulation S4. 


\section{Thème 7 - Risques côtiers}

Au niveau de la bouée DART 32412 (au large du Chili), le signal présente une très faible dispersion considérée comme négligeable. Celle-ci n’apparaît qu’à partir de la seconde bouée DART 43412 (au large du Mexique), à environ 7400 km. Parmi l'ensemble des simulations (et en considérant le décalage temporel induit par les autres formes de dispersion), la simulation S4 (équations de Boussinesq (2) avec une résolution de $2 \mathrm{~min}$, en bleu sur la figure 2) produit les signaux les plus proches de ceux enregistrés par les différentes bouées DART. On constate que la simulation S2 (équations de Saint-Venant (1) avec une résolution de $5 \mathrm{~min}$ ) produit des signaux proches de la simulation S4.

La dispersion fréquentielle calculée dans les différentes simulations (S1 à S4) peut être mise en évidence par les spectrogrammes des signaux au niveau des différentes bouées. La figure 3 représente ainsi le spectrogramme des séries temporelles mesurée (figure 3 (a)) et calculées (figures 3 (b), (c) et (d)) au large du Mexique entre 6 et 16 h après le séisme, que l'on compare à la dispersion fréquentielle théorique représentée en vert sur la figure 3. La simulation S1 (figure 3 (b)) n'induit que très peu de dispersion alors que la simulation S3 (figure 3 (d)) en induit trop. Seules les simulations S2 (figure 3 (c)) et S4 (figure 3 (e)) tendent à reproduire la dispersion fréquentielle physique de la figure 3 (a). Ceci permet donc de confirmer l'étude de ZHOU et al. (2012).

\section{Discussion}

Afin de mieux caractériser l'apport des équations de Boussinesq (2) par rapport aux équations de Saint-Venant (1), une analyse monodimensionnelle est menée. Elle permet notamment de simuler une série de cas en faisant varier le pas d'espace de $\Delta x=0.5 \mathrm{~km}$ à $\Delta x=20 \mathrm{~km}(2 \mathrm{~km} \sim 1 \mathrm{~min})$ afin de connaître la résolution minimale nécessaire pour une propagation trans-Pacifique.

Le canal utilisé a une profondeur de $4000 \mathrm{~m}$ et la vague initiale est similaire à celle du tsunami généré au large du Chili en 2010. Plus précisément, les formules d’Okada sont appliquées et conduisent à générer une arche dont la longueur atteint environ $100 \mathrm{~km}$ après quelques minutes de propagation. Ce cas correspond à une propagation faiblement non linéaire (l'amplitude de la vague est négligeable comparée à la profondeur) et faiblement dispersive $(\lambda / d=200000 / 4000 \sim 50)$, avec $\lambda$ la longueur d'onde et $d$ la profondeur. Les figures 4 et 5 représentent respectivement les vagues après 2000 et $10000 \mathrm{~km}$ de propagation.

Les solutions calculées par les modèles de Saint-Venant (figure 4 (a) et 5 (a)) et de Boussinesq (figure 4 (b) et 5 (b)) sont représentées pour trois pas d'espace, $\Delta x=1,5$, $10 \mathrm{~km}$.

Les surfaces d'eau calculées par Saint-Venant montrent que les résultats dépendent fortement du pas d'espace choisi en raison de la dispersion numérique associée au schéma. Des tests sur la résolution montrent que la convergence est obtenue pour des pas d'espace inférieurs ou égaux à $\Delta x=1 \mathrm{~km}$ environ. Les hautes fréquences, qui 


\section{XIV èmes Journées Nationales Génie Côtier - Génie Civil \\ Toulon, 29 juin au $1^{\text {er }}$ juillet 2016}

apparaissent pour des pas d'espace fins, ont pour origine une discontinuité de la surface de l'eau à l’instant initial.

(a)

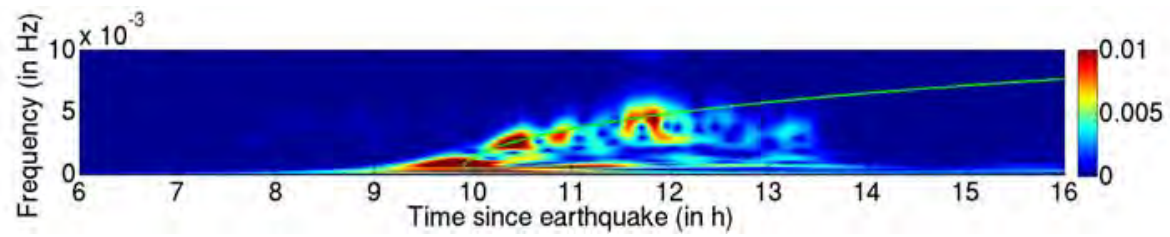

(b)

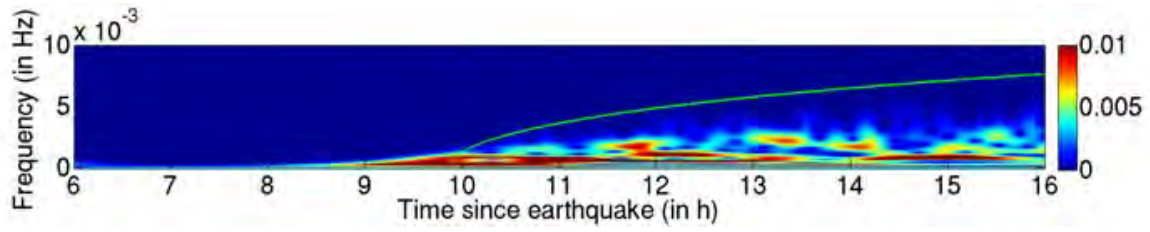

(c)

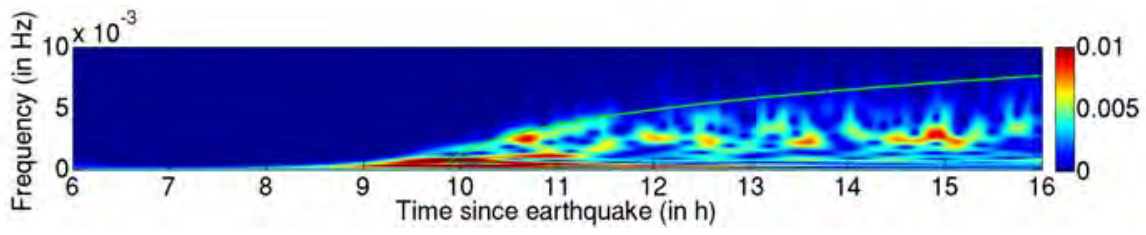

(d)
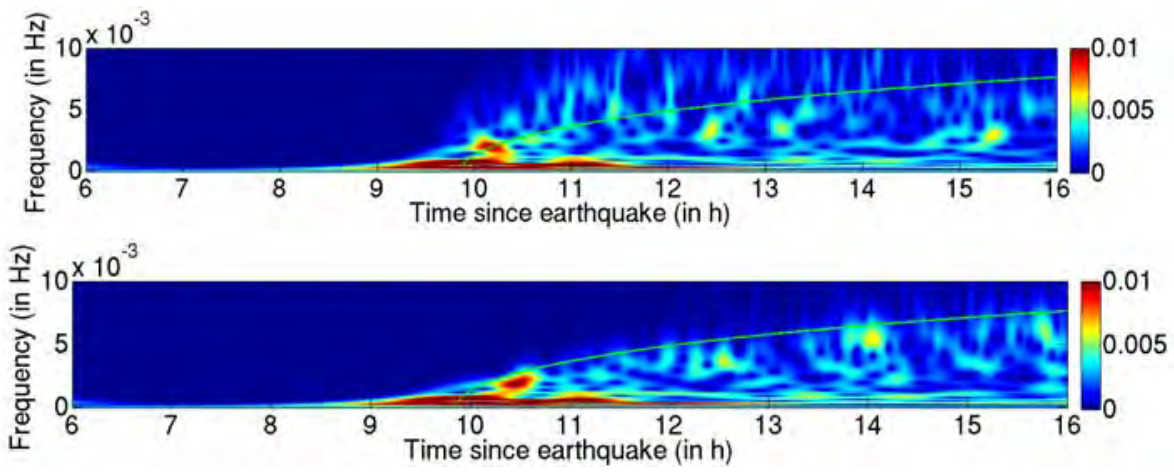

(e)

Figure 3. Spectrogrammes associés à la bouée DART 41413 située au large du Mexique pour le/la : (a) signal mesuré, (b) simulation S1, (c) simulation S2, (d) simulation S3, (e) simulation S4. La dispersion fréquentielle théorique est représentée par la courbe verte.

Les surfaces de l'eau calculées par le modèle de Boussinesq dépendent moins du maillage et une solution convergente est obtenue à partir d'un pas d'espace de $\Delta x=$ $5 \mathrm{~km}$ notamment lorsque la première vague est considérée.

En choisissant un pas d'espace $\Delta x=10 \mathrm{~km}$ égal à environ 2 fois la profondeur, la relation de dispersion du modèle Saint-Venant conduit à calculer une dispersion numérique proche de la dispersion fréquentielle physique. Les surfaces calculées par le modèle de Saint-Venant (figure 4 (a) et 5 (a)) sont alors proches de celles calculées par le modèle de Boussinesq (figure 4 (b) et 5 (b)) qui prend en compte la dispersion fréquentielle physique. Les tests de sensibilité confirment que ce résultat est indépendant de la longueur initiale de l'arche. En conclusion, ce cas permet de conclure que la dispersion fréquentielle est présente et peut être calculée par le modèle de Boussinesq à partir d'un pas d'espace de $\Delta x=5 \mathrm{~km}$. 


\section{Thème 7 - Risques côtiers}

Ces résultats permettent donc d'amener des éléments de comparaison à l'étude du tsunami chilien de 2010 pour laquelle les simulations S2 (équations de Saint-Venant (1) avec une résolution de ) et S4 (équations de Boussinesq (2) avec une résolution de ) permettaient de se rapprocher au mieux des hauteurs de vagues mesurées aux différentes bouées DART sélectionnées à travers le Pacifique.
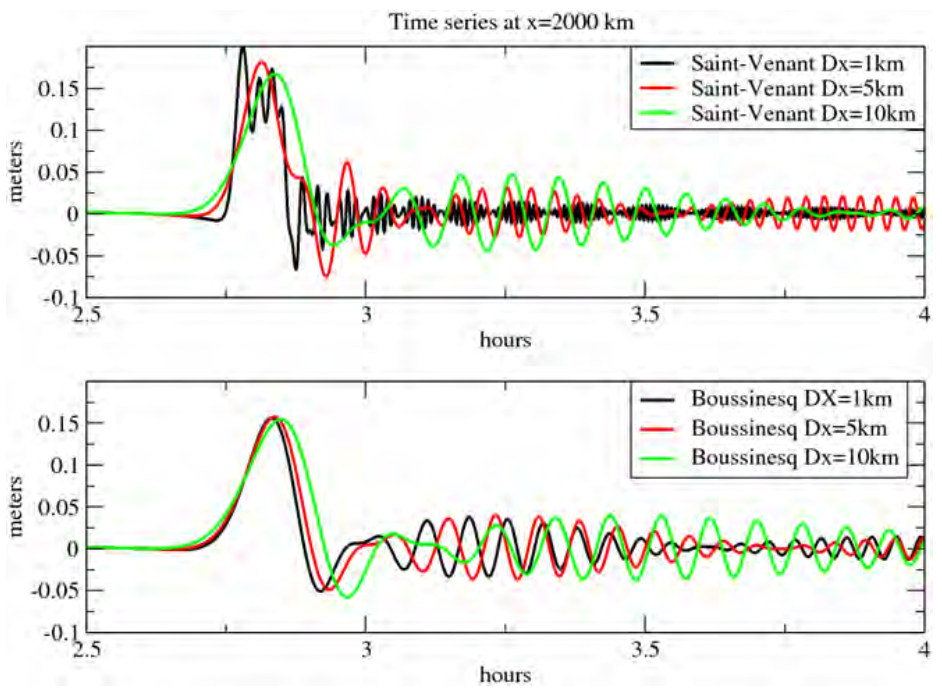

Figure 4. Propagation d'une vague en monodimensionnel par $4000 \mathrm{~m}$ de fond par les modèles de Saint-Venant (a) et Boussinesq (b). La figure représente la série temporelle à $2000 \mathrm{~km}$ de la source, pour des pas d'espace (en noir),

(en

$$
\text { rouge) et (envert). }
$$
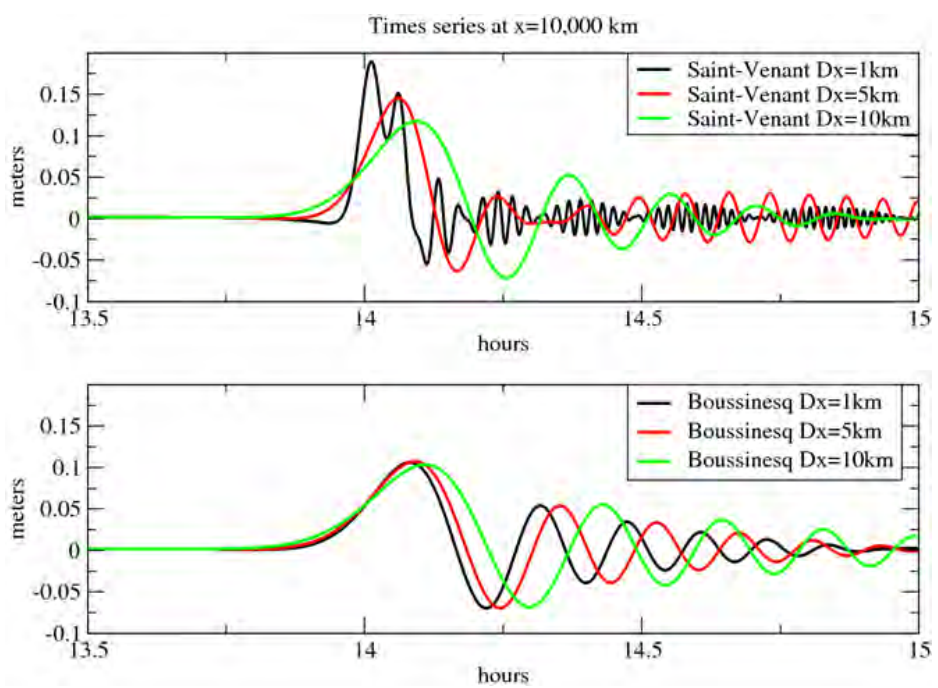

Figure 5. Propagation d'une vague en monodimensionnel par $4000 \mathrm{~m}$ de fond par les modèles de Saint-Venant (a) et Boussinesq (b). La figure représente la série temporelle à $10000 \mathrm{~km}$ de la source, pour des pas d'espace

$$
\text { rouge) et (envert). }
$$

(en noir), (en 


\section{XIV ${ }^{\text {èmes }}$ Journées Nationales Génie Côtier - Génie Civil \\ Toulon, 29 juin au $1^{\text {er }}$ juillet 2016}

\section{Conclusion}

Le tsunami généré par le séisme de 2010 de magnitude 8.8 a été affecté par la dispersion fréquentielle. Les simulations numériques réalisées ont permis de faire apparaître cette dispersion lors de la propagation du tsunami. Parmi les simulations réalisées, c'est la simulation (S4) impliquant les équations de Boussinesq qui se rapproche le plus des observations. La simulation (S2), considérant les équations de Saint-Venant et une grille de résolution 5 min, génère de la dispersion numérique qui se rapproche de la dispersion physique observée. Une étude paramétrique 1D a permis de réaliser des tests de convergence et de montrer que le modèle de Boussinesq converge à partir d'un pas d'espace de $5 \mathrm{~km}(2.5 \mathrm{~min} \sim 5 \mathrm{~km})$ et que la dispersion numérique induite par les équations de Saint-Venant peut se rapprocher de la dispersion fréquentielle du tsunami pour un pas d'espace de $10 \mathrm{~km}$.

Nous montrons également que la dispersion fréquentielle du tsunami a un impact non négligeable sur le temps d'arrivée, l'amplitude et la fréquence des vagues se propageant en champ lointain. Elle doit donc être prise en compte pour l'étude de l'amplification des vagues lorsque celles-ci atteignent les côtes, et notamment dans les zones habitées à risque.

Il est ainsi envisagé de propager le tsunami dans les baies de Nuku Hiva et de Hiva Oa en Polynésie Française, de Hilo à Hawaii et de Kamaïshi au Japon en améliorant la résolution de la bathymétrie pour ces zones, ce qui exige d'utiliser un système de grilles imbriquées. Ces modélisations permettront de comparer nos résultats avec les signaux enregistrés par les différents marégraphes situés dans ces baies. Enfin, les modélisations du tsunami généré par le séisme chilien de 2015 seront également analysées en termes de dispersion fréquentielle. Compte tenu de sa magnitude plus faible, la dispersion fréquentielle du tsunami sera plus marquée.

\section{Remerciements}

Nous tenons à remercier nos partenaires du projet TANDEM qui mettent à disposition leurs grilles de calcul et leurs données marégraphiques, et notamment le département de recherche en sismologie, météorologie et volcanologie de Tsukuba au Japon. Nous remercions également le centre de recherche sur les tsunamis de l'administration océanique et atmosphérique nationale (NOAA).

\section{Références bibliographiques}

GLIMSDAL S., PEDERSEN G. K., HARBITZ C. B., LØVHOLT F. (2013). Dispersion of tsunamis: does it really matter? Nat. Hazards Earth Syst. Sci., Vol. 13, pp 1507-1526. http://dx.doi.org/10.5194/nhess-13-1507-2013

GRILLI S.T., HARRIS J.C., TAJALI BAKHSH T.S., MASTERLARK T.L., KYRIAKOPOULOS C., KIRBY J.T., SHI F. (2012). Numerical Simulation of the 2011 
Tohoku Tsunami Based on a New Transient FEM Co-seismic Source: Comparison to Far- and Near-Field Observations. Pure and Applied Geophysics, Vol. 170(6), pp 1333-1359. http://dx.doi.org/10.1007/s00024-012-0528-y

HORRILLO J., KOWALIK Z., SHIGIHARA Y. (2006). Wave Dispersion Study in the Indian Ocean-Tsunami of December 26, 2004. Marine Geodesy, Vol. 29, pp 149-166. http://dx.doi.org/10.1080/01490410600939140

IOUALALEN M., ASAVANANT J., KAEWBANJAK N., GRILLI S. T., KIRBY J.T., WATTS P. (2007). Modeling the 26 December 2004 Indian Ocean tsunami: Case study of impact in Thailand. J. Geophys. Res., Vol. 112, C07024. http://dx.doi.org/10.1029/2006jc003850

JAMELOT A., REYMOND D. (2015). New tsunami Forecast Tools for the French Polynesia Tsunami Warning System Part II: Numerical Modelling and Tsunami Height Estimation. Pure and Applied Geophysics, Vol. 172 (3), pp 805-819. http://dx.doi.org/10.1007/s00024-014-0997-2

LØVHOLT F., PEDERSEN G., GISLER G. (2008). Oceanic propagation of a potential tsunami from the La Palma Island. J. Geophys. Res., Vol. 113, C09026. http://dx.doi.org/10.1029/2007jc004603

LØVHOLT F., KAISER G., GLIMSDAL G., SCHEELE L., HARBITZ C.B., PEDERSEN G. (2012). Modeling propagation and inundation of the 11 March 2011 Tohoku tsunami. Nat. Hazards Earth Sci. Vol. 12, pp 1017-1028. http://dx.doi.org/10.5194/nhess-12-1017-2012

REYMOND D., HYVERNAUD O., OKAL E. (2013). The 2010 and 2011 Tsunamis in French Polynesia: Operational Aspects and Field Surveys. Pure and Applied Geophysics, Vol 170(6-8), pp 1169-1187. http://dx.doi.org/10.1007/s00024-012-0485-5 SAITO T., MATSUZAWA T., OBARA K., BABA T. (2010). Dispersive tsunami of the 2010 Chile earthquake recorded by the high-sampling-rate ocean-bottom pressure gauges. Geo. Res. Let., Vol. 37, L23303. http://dx.doi.org/10.1029/2010gl045290

WATADA S., KUSUMOTO S., SATAKE K (2014). Traveltime delay and initial phase reversal of distant tsunamis coupled with the self-gravitating elastic Earth. Journal of Geophys. Res. Solid Earth, Vol. 119, pp 4287-4310. http://dx.doi.org/10.1002/2013JB010841 ZHOU H., MOORE C.W., WEI Y., TITOV V.V. (2011). A nested-grid Boussinesq-type approach to modelling dispersive propagation and runup of landslide-generated tsunamis. Nat. Hazards Earth Syst. Sci., Vol. 11, pp 2677-2697. http://dx.doi.org/10.5194/nhess-11-2677-2011

ZHOU H., WEI Y., TITOV V.V. (2012). Dispersive modeling of the 2009 Samoa tsunami. Geophysical Research Letters, Vol. 39, L16603. 